\title{
Non-Negative Matrix Factorization Based UKF Algorithm for Constant Modulus Signals in Adaptive Beamforming
}

\author{
Ranganathan Vignesh, K. A. Narayanankutty \\ (Amrita University), Coimbatore, India \\ Email: vigneshranganathan@gmail.com,ka_narayanankutty@yahoo.com
}

Department of Electronics and Communication Engineering, Amrita School of Engineering, Coimbatore, Amrita Vishwa Vidyapeetham

How to cite this paper: Vignesh, R. and Narayanankutty, K.A. (2016) Non-Negative Matrix Factorization Based UKF Algorithm for Constant Modulus Signals in Adaptive Beamforming. Open Journal of Antennas and Propagation, 4, 119-127.

http://dx.doi.org/10.4236/ojapr.2016.43009

Received: July 10, 2016

Accepted: August 19, 2016

Published: August 22, 2016

Copyright (c) 2016 by authors and Scientific Research Publishing Inc. This work is licensed under the Creative Commons Attribution International License (CC BY 4.0).

http://creativecommons.org/licenses/by/4.0/

(c) (i) Open Access

\section{Abstract}

Blind adaptive beamforming is getting appreciated for its various applications in contemporary communication systems where sources are statistically dependent or independent that are allowed to formulate new algorithms. Qualitative performance and time complexity are the main issues. In this paper, we propose a technique for constant modulus signals applying basic non-negative matrix factorization (BNMF) in blind adaptive beamforming environment. We compared the existing Unscented Kalman Filter based Constant Modulus Algorithm (UKF-CMA) with proposed NMF-UKF-CMA algorithm. We see there is a better improvement of sensor array gain, signal to interference plus noise ratio (SINR) and mean squared deviation (MSD) as the noise variance and the array size increase with reduced computational complexity with the UKF-CMA.

\section{Keywords}

Blind Adaptive Beamforming, NMF-UKF-CMA, Performance Comparison, UKF-CMA, MSD, SINR

\section{Introduction}

Adaptive blind beamforming plays an important role in the contemporary communication systems where it constantly tributes to the enhancement of the signals that tend to be received or transmitted. Adaptive beamforming is achieved through varying the tap weights assigned to each antenna at every time instant applying signal processing algorithm.

The weights are adjusted such that maximum array sensor gain is obtained with mi- 
nimal amount of residual error. On processing the beamforming signals, the computational complexity depends on the algorithm which works upon the signals. The recent UKF-CMA algorithm for blind beamforming application works quite well compared to other beamforming techniques such as Least Mean Squared-Constant Modulus Algorithm (LMS-CMA) and Recursive Least Mean Squared-Constant Modulus Algorithm (RLS-CMA) with higher computational complexity [1].

The UKF-CMA algorithm enabled in Gaussian conditions converges to optimal solution when measurement noise is considered. However, UKF-CMA with process noise results in sub-optimal solution [2] [3]. The CM criterion is incorporated into Weiner filter through which adaptability is achieved [2]. Generally, Constant Modulus (CM) cost functions with quadratic nature are very sensitive to array tap weights and can be minimized using Stochastic Gradient Descent methods (SGD) and the stability of SGD methods relatively depends on the step-size selected and thus results in slow rate of convergence [2].

An approximation of various $\mathrm{CM}$ algorithms is proposed. The computational cost of the Lagrangian formulated beamforming methods is higher over the regularized beamforming methods [4]. In unscented transform, the choice of sigma points is controlled by $\lambda$, which in turn linearises equal to the second order Gauss filter that results in optimal convergence of the solution [3] [5]. A new discriminant based non-negative matrix factorization algorithm is proposed for facial image characterization problems where discriminant analysis is based on the classification features [6].

A variant of NMF algorithm is proposed for blind source separation where it is a promising solution for spectral unmixing in hyper-spectral image processing and feature extraction [7]. Different methods of initialization are studied for NMF algorithm, where initialization plays an important role since decomposition is non-convex with many local minima [8].

\section{Non-Negative Matrix Factorization Algorithm}

Non-Negative Matrix Factorization (NMF), a relatively novel technique for dimensionality reduction, has been in the growing fast since its origin. It incorporates the non-negativity constraint and thus achieves the parts-based representation as well as enhancing the construe of the problem correspondingly [9] [10]. Some new algorithms for NMF are proposed for blind source separation application when sources are statistically dependent by imposing constraints to the matrix [11]. Multichannel NMF decomposition algorithms are proposed for blind audio source separation. More variants of NMF algorithms for blind sources separation techniques can be found in [12]-[14]. An extensive survey of NMF algorithms can be seen in [15]. In rectangular matrix, the solution is normally iterative and the steps normally require a $s \times b \times \min (s, b)$. In NMF, we make sure that the complexity is reduced to $s \times b \times t$, where $t$ is the rank of the matrix. This is achieved by factoring the matrix, as a product of 2 matrices, where first matrix acts as a set of basis vectors and other is positive definite. In quadratic problems, the coefficient matrix has to be positive-definite which 
is not true in general case, NMF forces the coefficient matrix to be positive-definite that results in closed-form solution.

Figure 1 describes about the flow of the algorithm.

The algorithm can be given as,

Initialize $\boldsymbol{U}_{o}, \boldsymbol{V}_{o}$ and $m=0$

for

$\boldsymbol{U}_{m+1} \leftarrow \boldsymbol{U}_{m}$ o $\frac{\left[\boldsymbol{Z} \boldsymbol{V}_{m}\right]}{\left[\boldsymbol{U}_{m} \boldsymbol{V}_{m}^{\mathcal{H}} \boldsymbol{V}_{m}\right]}$

$\boldsymbol{V}_{m+1} \leftarrow \boldsymbol{V}_{m} \quad \mathrm{o} \frac{\left[\boldsymbol{Z}^{\mathcal{H}} \boldsymbol{U}_{m+1}\right]}{\left[\boldsymbol{V}_{m} \boldsymbol{U}_{m+1}^{\mathcal{H}} \boldsymbol{U}_{m+1}\right]}$

$m \leftarrow m+1$

end

Where $\mathbf{Z} \in \mathbb{C}^{s \times b}, \boldsymbol{U}_{m} \in \mathbb{C}_{+}^{s \times t}, \boldsymbol{V}_{m} \in \mathbb{C}_{+}^{b \times t}$ are non-negative matrices and the reduced rank $t$ is given by $t<\min (s, b)$ where $(s, b, t) \in \mathbb{R}_{+}$.

In this paper, we have reduced the computational complexity of UKF-CMA algorithm by reducing dimensionality of the matrix computation, which is achieved through the non-negative matrix factorization.

Note: Notations followed in the paper are bold small letters are vector. Capital letters are matrix.

\section{Beamforming Model}

Consider a linear array of size $L$ of uniform spacing $d \leq \frac{\lambda}{2}$ and $n$ is the number of source signals (interference and desired signals). The signal output of an adaptive beamformer is represented as [1],

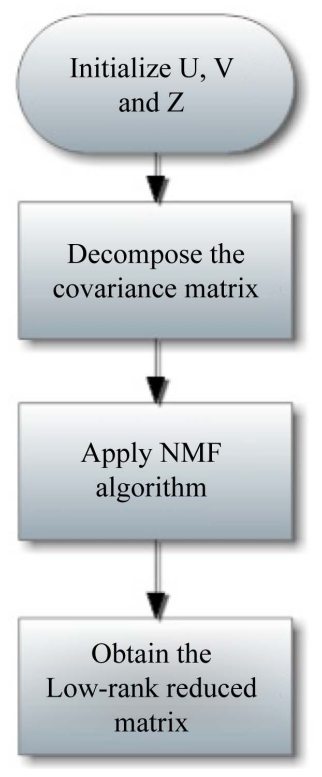

Figure 1. Flowchart of NMF-UKF-CMA algorithm. 


$$
\mathbf{z}_{m}=\boldsymbol{u}_{m}^{\mathcal{H}} \boldsymbol{w}
$$

The input signal vector $\boldsymbol{u}_{m} \in \mathbb{C}^{L \times 1}$ as,

$$
\boldsymbol{u}_{m}=\boldsymbol{D} \boldsymbol{s}_{m}+\boldsymbol{n}_{m}
$$

where $\boldsymbol{w} \in \mathbb{C}^{L \times 1}, \boldsymbol{s}_{m} \leftarrow\left[s_{1, m} ; s_{2, m} ; \cdots ; s_{n, m}\right]$ is the source signal vector whose first element is desired signal and remaining elements of the vector are made as interference signals, $\boldsymbol{n}_{m} \in \mathbb{C}^{L \times 1}$ is circular complex Gaussian noise at $m$-th time instant.

The spatial signature matrix $\boldsymbol{D}=\left[\begin{array}{llll}\Theta_{1} & \Theta_{2} & \cdots & \Theta_{L}\end{array}\right]$, Where $\boldsymbol{D} \in \mathbb{C}^{L \times k}$, $\Theta=\left[\begin{array}{llll}1 e^{-j \gamma} & 1 e^{-j 2 \gamma} & \cdots & 1 e^{-j(L-1) \gamma}\end{array}\right]^{\mathcal{H}}, \gamma=k_{o} d \sin \left(\theta_{j}\right), \gamma$ is the array phase function, $k_{o}$ is the phase constant, $d$ is the array spacing, $\theta_{i}$ is $i^{\text {th }}$ element of the AOA vector $\theta \in \mathbb{R}^{L \times 1}$.

The Constant Modulus (CM) cost function for adaptive beamforming problem can be formulated as

$$
\min _{\boldsymbol{w}} \boldsymbol{J}_{p, q}(\boldsymbol{w})=\left[\left|\boldsymbol{u}_{m}^{\mathcal{H}} \boldsymbol{w}\right|^{p}-\zeta\right]^{q}
$$

where $p>0, q>0$ and $\zeta$ is the signal modulus of the desired signal $\boldsymbol{s}_{m}$, which is a known a priori. As stated, the optimization problem is non-convex and non-linear.

\section{Algorithm Formulation}

The constant modulus criterion in (3) assumes that the unknown system model $\boldsymbol{f}_{m}$ for the input signal $\boldsymbol{u}_{m}$ is equal to the constant modulus of the desired signal $\zeta$ in (5).

$$
\begin{aligned}
& \boldsymbol{f} \leftarrow \boldsymbol{f}_{m \mid m-1} \\
& \zeta \leftarrow\left|\boldsymbol{u}_{m}^{\mathcal{H}} \boldsymbol{f}_{m \mid m-1}\right|^{p}
\end{aligned}
$$

The final state space model is obtained by incorporating process noise $\boldsymbol{q}_{m}$. Since initial received signal is unknown, so we take it as noise $\boldsymbol{v}_{m}$ adding to the model in (7). Applying the non-linearity $g()=.||^{p}$ in (8).

$$
\begin{aligned}
& \tilde{\boldsymbol{f}}_{m} \leftarrow \boldsymbol{A}_{m} \tilde{\boldsymbol{f}}_{m \mid m-1}+\boldsymbol{q}_{m \mid m-1} \\
& \zeta \leftarrow \tilde{\boldsymbol{f}}_{m \mid m-1}(L+1)+v_{m} \\
& \tilde{\boldsymbol{Z}}_{m} \leftarrow \mathrm{g}\left(\tilde{\boldsymbol{f}}_{m \mid m-1}\right) \\
& \boldsymbol{A}_{m} \leftarrow\left[\begin{array}{ll}
\boldsymbol{I} & 0 \\
\boldsymbol{u}_{m} & 0
\end{array}\right] \quad \boldsymbol{f}_{m} \leftarrow\left[\begin{array}{l}
\boldsymbol{f}_{m-1} \\
z_{m-1}
\end{array}\right]
\end{aligned}
$$

where $\boldsymbol{A}_{m} \in \mathbb{C}^{L+1 \times L+1}, \boldsymbol{f}_{m} \in \mathbb{C}^{L+1 \times 1}$ and $\boldsymbol{q}_{m} \in \mathbb{C}^{L+1 \times 1}$ is the process noise.

In (10) $\quad \tilde{\mathbf{Z}}_{m} \approx \tilde{\mathbf{Z}}$ is approximated by non-negative matrix factorization.

$$
\begin{gathered}
\tilde{\boldsymbol{Z}} \leftarrow \boldsymbol{U}_{m} \boldsymbol{V}_{m}^{\mathcal{H}} \\
\boldsymbol{U}_{m} \boldsymbol{V}_{m}^{\mathcal{H}} \rightarrow \tilde{\tilde{Z}}_{m}
\end{gathered}
$$

where $\tilde{\tilde{\mathbf{Z}}}_{m} \in \mathbb{C}^{s \times b}, \boldsymbol{U}_{m} \in \mathbb{C}_{+}^{s \times t}, \boldsymbol{V}_{m} \in \mathbb{C}_{+}^{b \times t}$ are non-negative matrices and the reduced rank $t$ is given by $t<\min (s, b)$ where $(s, b, t) \in \mathbb{R}_{+}$. In the algorithm formulation, we 
ignore the process noise $\boldsymbol{q}_{m}$ on including leads to suboptimal solution.

\section{Proposed NMF-UKF-CMA Algorithm}

The proposed NMF-UKF-CMA algorithm is as follows,

Input: $\boldsymbol{u}_{m}, \boldsymbol{w}_{m}, L, \alpha, \beta, \kappa, \lambda, \boldsymbol{w}_{m}, \boldsymbol{w}_{c}$

Initialize: $\alpha \leftarrow 1, \quad \beta \leftarrow 2, \quad \kappa \leftarrow 0, \quad n_{w} \leftarrow L+1, \quad \lambda \leftarrow \alpha^{2}\left(n_{w}+\kappa-1\right)-n_{w}+2$,

$\boldsymbol{w}_{m} \leftarrow \frac{1}{2\left(n_{w}+\lambda\right)}$ ones $\left(n_{w}, n_{w}\right), \quad \boldsymbol{w}_{c} \leftarrow \boldsymbol{w}_{m}, \quad \boldsymbol{w}_{m}(1) \leftarrow \frac{\lambda}{\lambda+n_{w}}$,

$\boldsymbol{w}_{c}(1) \leftarrow \frac{\lambda}{\left(\lambda+n_{w}\right)}+1-\alpha^{2}+\beta$ then initialize the unscented Kalman filter with

$\hat{\boldsymbol{w}}_{0} \leftarrow[\mathbf{1} ; \mathbf{0}], \boldsymbol{P}_{0} \leftarrow 1 / n_{w} \boldsymbol{I}$ of size $n_{w} \times n_{w}$,

for $m \geq 1$

do

Compute and update

- Extract the sigma points $\mathrm{W}_{m \mid m-1} \in \mathbb{C}^{n_{w} \times 2 n_{w}+1}$ as

$$
\mathrm{W}_{m \mid m-1} \leftarrow\left[\begin{array}{lll}
\hat{\boldsymbol{w}}_{m-1} & \hat{\boldsymbol{w}}_{m-1}+\sqrt{\left(n_{w}+\lambda\right) \boldsymbol{P}_{m-1}} & \hat{\boldsymbol{w}}_{m-1}-\sqrt{\left(n_{w}+\lambda\right) \boldsymbol{P}_{m-1}}
\end{array}\right]
$$

where $\boldsymbol{w} \in \mathbb{R}^{n_{w} \times 1}$ is an initial weight vector.

- Extract matrix $\boldsymbol{A}_{m}$ for the input signal $\boldsymbol{u}_{m}$ as

$$
\boldsymbol{A}_{m} \leftarrow\left[\begin{array}{cc}
I & 0 \\
u_{m} & 0
\end{array}\right]
$$

and then get the sigma points $\mathrm{W}_{m \mid m-1}^{-} \in \mathbb{C}^{n_{W} \times 2 n_{W}+1}$ for the updated state as

$$
\mathrm{W}_{m \mid m-1}^{-} \leftarrow \boldsymbol{A}_{m} \mathrm{~W}_{m \mid m-1}
$$

where $\boldsymbol{A}_{m} \in \mathbb{C}^{n_{w} \times n_{w}}$.

- Extract the posteriori estimate $\hat{\boldsymbol{w}}_{m \mid m-1}^{-} \in \mathbb{C}^{n_{w} \times 1}$ as

$$
\hat{\boldsymbol{w}}_{m}^{-} \leftarrow \sum_{j=1}^{2 n_{w}+1} \boldsymbol{w}_{m}^{j} \mathrm{~W}_{m \mid m-1}^{-j}
$$

where $j$ denotes the $j$-th column vector for $\mathrm{W}_{m \mid m-1}^{-}$and $j$-the element for vector $\boldsymbol{w}_{m} \in \mathbb{C}^{n_{w} \times n_{w}}$.

- Extract the sigma priori covariance $\boldsymbol{P}_{m}^{-} \in \mathbb{C}^{n_{w} \times n_{w}}$ as

$$
\boldsymbol{P}_{m}^{-} \leftarrow \sum_{j=1}^{2 n_{w}+1} \boldsymbol{w}_{c}^{j}\left(\mathrm{~W}_{m \mid m-1}^{-j}-\boldsymbol{w}_{m}^{-j}\right)\left(\mathrm{W}_{m \mid m-1}^{-j}-\boldsymbol{w}_{m}^{-j}\right)^{\mathcal{H}}
$$

where $j$ is the $j$-th column vector for $\boldsymbol{w}_{m}^{-}$and $j$-th element for vector $\boldsymbol{w}_{c}$.

- Extract the sigma points $Z_{m \mid m-1}^{-} \in \mathbb{C}^{n_{w} \times 2 n_{w}+1}$ through non-linear function as

$$
\mathbf{Z}_{m \mid m-1}^{-} \leftarrow \mathrm{g}\left(\mathrm{W}_{m \mid m-1}^{-j}\right)
$$

where $\mathrm{g}(.) \leftarrow|\cdot|^{p}$ for each element of the $j$-th column vector for $\mathrm{W}_{m \mid m-1}^{-}$for $j \leftarrow 1,2, \cdots, 2 n_{w}+1$.

- The output sigma points are approximated using non-negative matrix factorization algorithm as $\tilde{\mathbf{Z}}_{m \mid m-1}^{-} \in \mathbb{C}^{n_{w} \times 2 n_{w}+1}$ 


$$
\tilde{\mathbf{Z}}_{m \mid m-1}^{-} \leftarrow \boldsymbol{U}_{m \mid m-1} \boldsymbol{V}_{m \mid m-1}^{\mathcal{H}}
$$

where $\boldsymbol{U}_{m} \in \mathbb{C}_{+}^{n_{w} \times t_{w}}, \quad \boldsymbol{V}_{m} \in \mathbb{C}_{+}^{2 n_{w}+1 \times t_{w}}$ are non-negative matrices and reduced rank $t_{w}<\min \left(n_{w}, 2 n_{w}+1\right)$.

- Applying the output sigma points to extract the estimated output $\hat{\mathbf{z}}_{m \mid m-1}^{-} \in \mathbb{C}^{n_{w} \times 1}$ as

$$
\hat{\mathbf{Z}}_{m \mid m-1}^{-} \leftarrow \sum_{j=1}^{2 n_{w}+1} \boldsymbol{w}_{m}^{j} \tilde{\mathbf{Z}}_{m \mid m-1}^{j}
$$

- The obtained crosscovariance matrix $C_{m m} \in \mathbb{C}^{n_{w} \times 1}$ as

$$
\boldsymbol{C}_{m m} \leftarrow \sum_{j=1}^{2 n_{w}+1} \boldsymbol{w}_{c}^{j}\left(\mathbf{W}_{m \mid m-1}^{-j}-\boldsymbol{w}_{m}^{-}\right)\left(\tilde{\mathbf{Z}}_{m \mid m-1}^{-j}-\hat{\mathbf{z}}_{m \mid m-1}^{-}\right)^{\mathcal{H}}
$$

- The obtained autocovariance $\boldsymbol{R}_{m m}$ as

$$
\boldsymbol{R}_{m m} \leftarrow \sum_{j=1}^{2 n_{w}+1} \boldsymbol{w}_{c}^{j}\left(\tilde{\boldsymbol{Z}}_{m \mid m-1}^{-j}-\hat{\mathbf{Z}}_{m}^{-}\right)\left(\tilde{\mathbf{Z}}_{m \mid m-1}^{-j}-\hat{\mathbf{Z}}_{m}^{-}\right)^{\mathcal{H}}+\sigma_{v}^{2}
$$

where $\sigma_{v}^{2}=\frac{1}{n_{w}-1} \sum_{j=1}^{n_{w}-1} \hat{\mathbf{z}}_{m \mid m-1}^{-j}$

- Now apply the Kalman innovation matrix and the update formulas as

$$
\begin{aligned}
& \boldsymbol{K}_{m} \leftarrow \boldsymbol{C}_{m m} \boldsymbol{R}_{m m}^{-1} \\
& \hat{\boldsymbol{w}}_{m} \leftarrow \hat{\boldsymbol{w}}_{m}^{-}+\boldsymbol{K}_{m}\left(\zeta-\hat{\boldsymbol{z}}_{m}^{-}\right)^{*} \\
& \boldsymbol{P}_{m} \leftarrow \boldsymbol{P}_{m \mid m-1}^{-}-\boldsymbol{K}_{m} \boldsymbol{R}_{m m} \boldsymbol{K}_{m}^{-1}
\end{aligned}
$$

- Update the optimal weight vector $\boldsymbol{w} \leftarrow \boldsymbol{w}_{m}^{-}\left(1: n_{w}-1,1\right)$. end

\section{Simulation and Results}

In this section, the performance of NMF-UKF-CMA algorithm is compared with existing UKF-CMA algorithm. An uniform linear array of length $L=20$ and of spacing $d=\lambda / 2$ for simulation. The constant modulus signals are generated by Minimum Shift Keying (MSK) Modulation scheme with unity modulus and the interference plus noise signal were set as Gaussian distributed random variables with mean, 0 and noise variance of 1 . An uniform distribution of $-\pi$ to $\pi$ is followed for phase. The desired direction of arrival is set as $10^{\circ}$ and for interference signals are set as $25^{\circ},-30^{\circ}$ and $-45^{\circ}$. The CMA criterion is chosen as $p=1$ and $q=2$ in the simulations, for which we achieve optimal signal-to-interference-plus-noise ratio (SINR). In addition to SINR in $\mathrm{dB}$, Mean Square Deviation (dB) and Array Sensor Gain (dB) are the parameters, also used to estimate the performance of the array algorithms. MSD is defined as $20 \log _{10}\left(\left|s_{m}-z_{m}\right| /\left|s_{m}\right|\right)$. The value of $v$ is set as 0.75 in all the simulations. The plots simulated are stochastic averages of 500 independent simulations.

\section{Simulation-1}

In Simulation-1, The interference plus noise signal of variance $\left(\sigma_{n}^{2}\right)$ is set as 0.1 . From Figure 2, NMF-UKF-CMA algorithm has improved gain and grating lobe suppression compared to UKF-CMA. Lesser mean square deviation for NMF-UKF- 

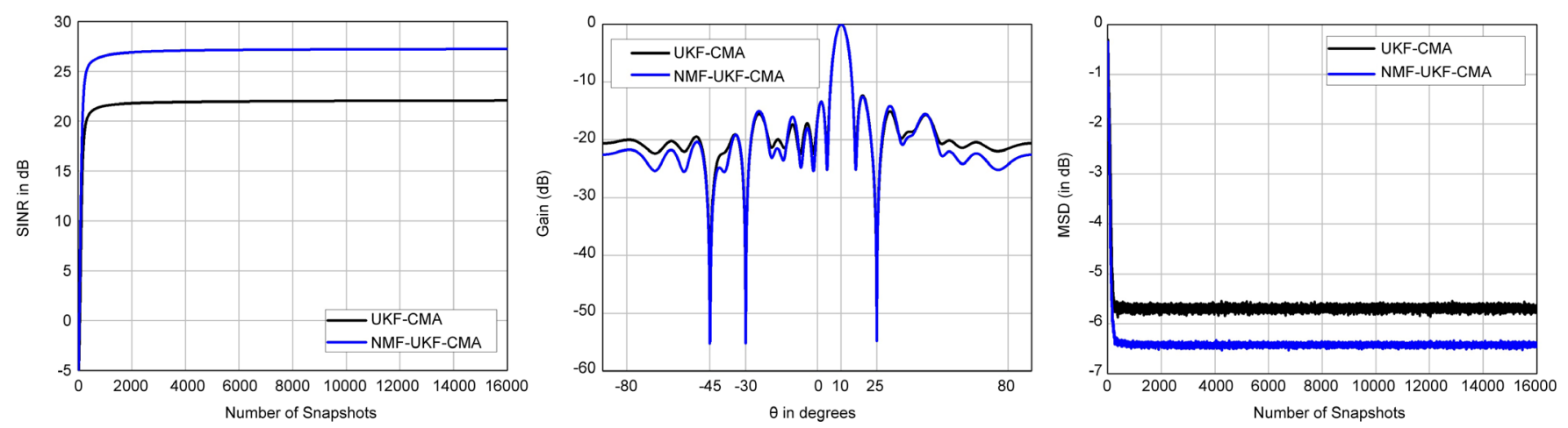

Figure 2. Simulation 1: SINR in dB (left), Array Sensor Gain in dB (center), MSD in dB (right) with $\sigma_{n}^{2} \leftarrow 0.1$.

CMA for the given noise variance to the UKF-CMA. The proposed NMF-UKF-CMA algorithm attains much better SINR compared with UKF-CMA as the sample size increases. The convergence of NMF-UKF-CMA algorithm gives better attenuation of interferences compared to UKF-CMA and it converges within the limited sample space.

\section{Simulation-2}

In Simulation-2,The interference plus noise signal of variance $\left(\sigma_{n}^{2}\right)$ is set as 0.0316 . From Figure 3, We achieve similar results compared to UKF-CMA as the noise variance decreases. We could observe betterment of proposed algorithm MSD compared to UKF-CMA. The SINR values of NMF-UKF-CMA algorithm closely follow the UKF-CMA algorithm.

\section{Simulation-3}

In Simulation-3, The number of antennas $L$ in the array is increased to 60 and remaining parameters are set as in simulation 1 . From Figure 4, we achieve an increased SINR for NMF-UKF-CMA algorithm as the number of antenna is increased.

\section{Simulation-4}

In Simulation-4, The number of sources $M$ is increased to 7 , interference's added in the direction of $35^{\circ}, 55^{\circ},-55^{\circ}$ and the number of antennas $L$ is decreased to 4 and $p$ is set as 0.5 for the simulation are performed. From Figure 5, As seen, there is degradation in SINR as the number of sources increased. The similarity in performance can be seen for NMF-UKF-CMA and UKF-CMA as the number of sources increased.

\section{Conclusion}

A technique for dimensionality reduction and compression of cross-covariance matrix is achieved through NMF Algorithm, found to be more effective in beamforming. NMF achieves superiority over the classic low rank reduction algorithms such as PCA and LDA by imposing purely additive constraint or positivity criteria on the matrix. The initialization and the determination of number of basis vectors add to faster convergence of the solution. By incorporating the technique in to a adaptive blind beamforming problem, our proposed NMF-UKF-CMA algorithm has better performance compared to UKF-CMA algorithm. On close observation, as the number of antennas in the array and noise variance increases, we achieve better Sensor Array Gain, Signal 

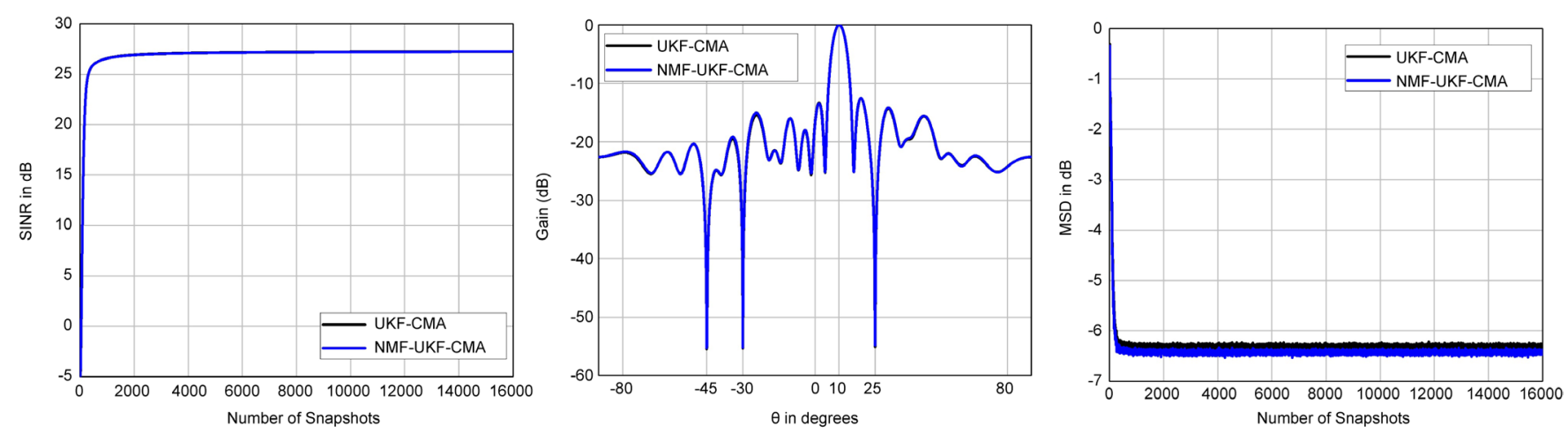

Figure 3. Simulation 2: SINR in dB (left), Array Sensor Gain in dB (center), MSD in dB (right) with $\sigma_{n}^{2}=0.0316$.

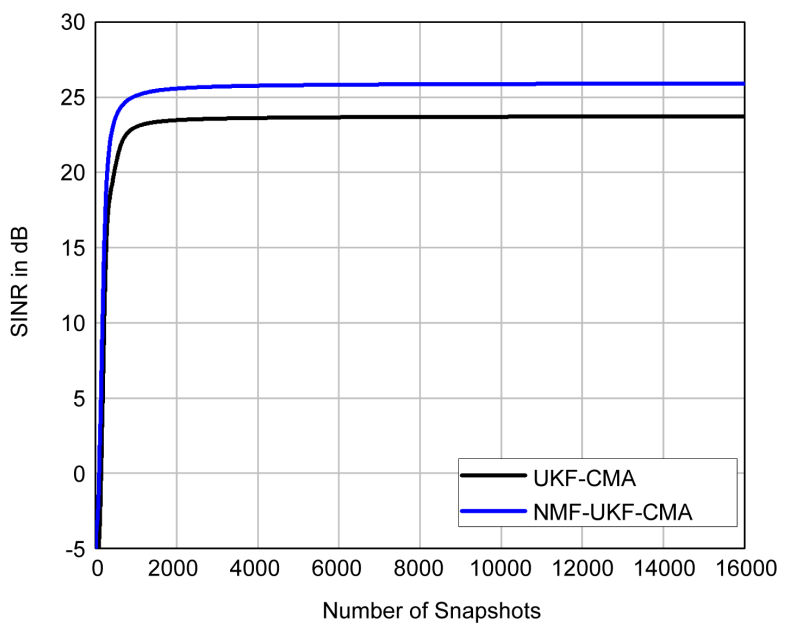

Figure 4. Simulation 3: SINR for $M=4, L=60$ and $\sigma_{n}^{2}=0.1$.

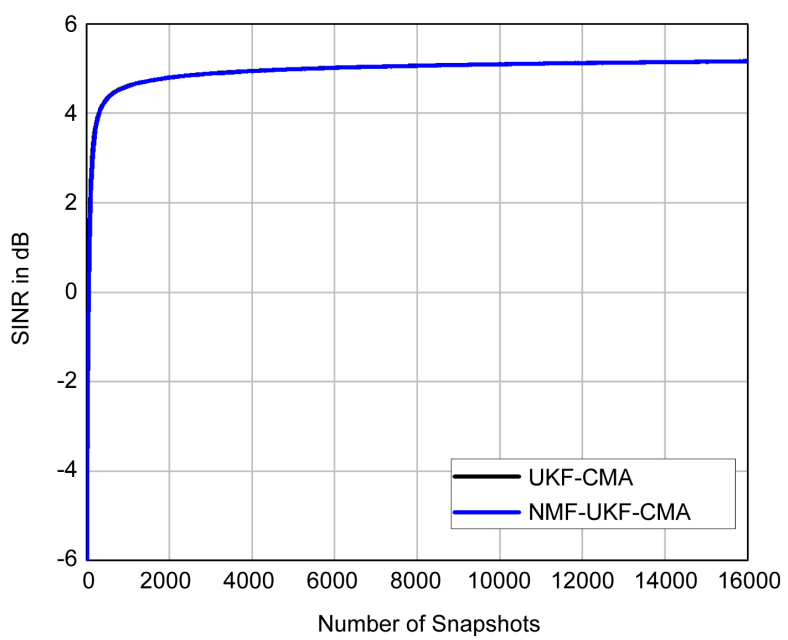

Figure 5. Simulation 4: SINR for $M=7, L=4$ and $\sigma_{n}^{2}=0.1$.

to Interference plus Noise Ratio and Mean Squared Deviation with reduced computational complexity. 


\section{References}

[1] Bhotto, Md.Z.A. and Bajic, I.V. (2015) Constant Modulus Blind Adaptive Beamforming Based on Unscented Kalman Filtering. IEEE Signal Processing Letters, 22, 474-478.

[2] Triechler, J.H. and Agee, B.G. (1983) A New Approach to Multipath Correction of Constant Modulus Signals. IEEE Transactions on Acoustics, Speech, and Signal Processing, 31, 459472.

[3] Julier, S.J. and Uhlmann, J.K. (2004) Unscented Filtering and Nonlinear Estimation. Proceedings of the IEEE, 92, 401-422. http://dx.doi.org/10.1109/JPROC.2003.823141

[4] J Li, J. and Stoica, P. (2005) Robust Adaptive Beamforming. Wiley, Hoboken.

[5] Julier, S. J. (2002) The Scaled Unscented Transformation. Proceedings of the 2002 American Control Conference, 6, 4555-4559. http://dx.doi.org/10.1109/acc.2002.1025369

[6] Kotsia, I., Zafeiriou, S. and Pitas, I. (2007) A Novel Discriminant Non-Negative Matrix Factorization Algorithm with Applications to Facial Image Characterization Problems. IEEE Transactions on Information Forensics and Security, 2, 3. http://dx.doi.org/10.1109/TIFS.2007.902017

[7] Rajabi, R. and Ghassemian, H. (2015) Spectral Unmixing of Hyperspectral Imagery Using Multilayer NMF. IEEE Geoscience and Remote Sensing Letters, 12, 38-42. http://dx.doi.org/10.1109/LGRS.2014.2325874

[8] Grais, E.M. and Erdogan, H. (2013) Initialization of Nonnegative Matrix Factorization Dictionaries for Single Channel Source Separation. Signal Processing and Communications Applications Conference (SIU), Haspolat, 24-26 April 2013, 1-4. http://dx.doi.org/10.1109/siu.2013.6531172

[9] Lee, D.D. and Seung, H.S. (1999) Learning the Parts of Objects by Non-Negative Matrix Factorization. Nature, 401, 788-791. http://dx.doi.org/10.1038/44565

[10] Lee, D.D. and Sebastian, S.H. (2001) Algorithms for Non-Negative Matrix Factorization. Advances in Neural Information Processing Systems 13, MIT Press, Cambridge, 556-562.

[11] Cichocki, A., Zdunek, R. and Amari, S. (2006) New Algorithms for Non-Negative Matrix Factorization in Applications to Blind Source Separation. 2006 IEEE International Conference on Acoustics Speech and Signal Processing Proceedings, Toulouse, 14-19 May 2006, V-V. http://dx.doi.org/10.1109/ICASSP.2006.1661352

[12] Ozerov, A. and Fevotte, C. (2009) Multichannel Nonnegative Matrix Factorization in Convolutive Mixtures. With Application to Blind Audio Source Separation. IEEE International Conference on Acoustics, Speech and Signal Processing, Taipei, 19-24 April 2009, 31373140. http://dx.doi.org/10.1109/icassp.2009.4960289

[13] Yang, Z., Zhou, G., Ding, S. and Xie, S. (2010) Blind Source Separation by Nonnegative Matrix Factorization with Minimum-Volume Constraint. International Conference on Intelligent Control and Information Processing (ICICIP), Dalian, 13-15 August 2010, 117-119. http://dx.doi.org/10.1109/icicip.2010.5565228

[14] Krbz, S. and Gunsel, B. (2012) Perceptually Weighted Non-Negative Matrix Factorization for Blind Single-Channel Music Source Separation. 21st International Conference on Pattern Recognition (ICPR), Tsukuba, 11-15 November 2012, 226-229.

[15] Wang, Y.X. and Zhang, Y.J. (2013) Nonnegative Matrix Factorization: A Comprehensive Review. IEEE Transactions on Knowledge and Data Engineering, 25, 1336-1353. http://dx.doi.org/10.1109/TKDE.2012.51 
Submit or recommend next manuscript to SCIRP and we will provide best service for you:

Accepting pre-submission inquiries through Email, Facebook, LinkedIn, Twitter, etc. A wide selection of journals (inclusive of 9 subjects, more than 200 journals)

Providing 24-hour high-quality service

User-friendly online submission system

Fair and swift peer-review system

Efficient typesetting and proofreading procedure

Display of the result of downloads and visits, as well as the number of cited articles

Maximum dissemination of your research work

Submit your manuscript at: http://papersubmission.scirp.org/ 